Copyright 2008 Society of Photo-Optical Instrumentation Engineers. This paper was published in Proc. SPIE 7020 and is made available as an electronic reprint with permission of SPIE. One print or electronic copy may be made for personal use only. Systematic or multiple reproduction, distribution to multiple locations via electronic or other means, duplication of any material in this paper for a fee or for commercial purposes, or modification of the content of the paper are prohibited.

\title{
Cryogenic magnetic shielding for SCUBA-2
}

\author{
Matthew Hollister ${ }^{a}$, Helen McGregor ${ }^{b}$, Adam Woodcraft ${ }^{a, b}$, Dan Bintley ${ }^{c}$, Michael MacIntosh $^{b}$ \\ and Wayne Holland ${ }^{b}$ \\ ${ }^{a}$ SUPA, Institute for Astronomy, Royal Observatory, Edinburgh, EH9 3HJ, UK; \\ ${ }^{b}$ UK Astronomy Technology Centre, Royal Observatory, Edinburgh, EH9 3HJ, UK; \\ ${ }^{c}$ Joint Astronomy Centre, 660 N. A'ohoku Place, Hilo, HI 96720, USA
}

\begin{abstract}
SCUBA-2 is a new wide-field submillimeter continuum instrument being commissioned on the James Clerk Maxwell Telescope on Mauna Kea in Hawaii. SCUBA-2 uses large-scale arrays of superconducting bolometers with SQUID- (superconducting quantum interference device) based multiplexing and amplification. The sensitivity of the devices that compose the detector arrays to magnetic fields is such that magnetic shielding, consisting of superconducting and high-permeability materials, was fitted to the detector enclosure at $1 \mathrm{~K}$ to reduce the magnetic field strength at the focal plane. This paper describes the design and construction of the cryogenic shielding, and presents verification measurements. The shielding performance was found to meet the instrument requirements, and compared well to the modelled results.
\end{abstract}

Keywords: SCUBA-2, Submillimeter bolometer array, Transition edge sensor, Superconducting quantum interference device, Magnetic shielding

\section{INTRODUCTION}

The submillimetre common-user bolometer array-2 (SCUBA-2) is a widefield survey camera presently being commissioned on the James Clerk Maxwell Telescope on Mauna Kea in Hawaii. SCUBA-2 images at 450 and $850 \mu \mathrm{m}$ using large-scale arrays of superconducting bolometers, with time-division multiplexing and cryogenic amplification provided by superconducting quantum interference devices (SQUIDs). More detailed descriptions of the instrument and science goals may be found elsewhere. ${ }^{1}$

The detector arrays must be well shielded against magnetic fields in order to function correctly and to meet the performance requirements of the SCUBA-2 instrument. The SQUIDs used to multiplex and amplify the signals from the detector pixels are extremely sensitive magnetometers. The presence of a strong AC magnetic field will modulate the critical currents of the SQUIDs. At best, this will appear as an additional modulation in the output from the devices. In a stronger field, the devices could be driven into the normal state for part of the cycle, effectively rendering the SQUIDs useless. The SQUID amplifiers, essentially a chain of $\sim 100 \mathrm{dc}$ SQUIDs in series, ${ }^{2}$ are far more sensitive to the field effects than the SQUID multiplexers as the presence of a strong field could induce phase differences along the series array, impairing the amplification of the signal from the multiplexer. Furthermore, one of the factors that control the sensitivity of the TES detectors is the width of the transition between the normal and superconducting states. Although the devices have been designed with a specific transition width, the presence of an AC or DC magnetic field will broaden this transition, potentially reducing the sensitivity of the detectors. The field requirements at the focal plane were that the magnetic field normal to the detector arrays must not exceed $100 \mathrm{nT}$ (the SQUIDs and TESs are planar structures, and hence have minimal sensitivity to tangential fields). Measurements of the magnetic field strengths at the telescope indicated that the field strength was approximately $0.15 \mathrm{mT}$. The shielding was therefore required to provide a minimum attenuation of 1500 .

Prior to the construction of the shielding, extensive modelling of the shield performance using a finiteelement method was employed in order to develop the shielding design efficiently. This work has been described previously $^{3}$ (hereafter Reference I) and the key results are reproduced in $§ 3$. The final design developed from the modelling process used a combination of high-permeability shielding at ambient temperature, and a combination

Further author information: (Send correspondence to M.H.) e-mail: matt.hollister@physics.org

Millimeter and Submillimeter Detectors and Instrumentation for Astronomy IV edited by William D. Duncan, Wayne S. Holland, Stafford Withington, Jonas Zmuidzinas Proc. of SPIE Vol. 7020, 702023, (2008) · 0277-786X/08/\$18 · doi: 10.1117/12.787795

Proc. of SPIE Vol. 7020 702023-1 

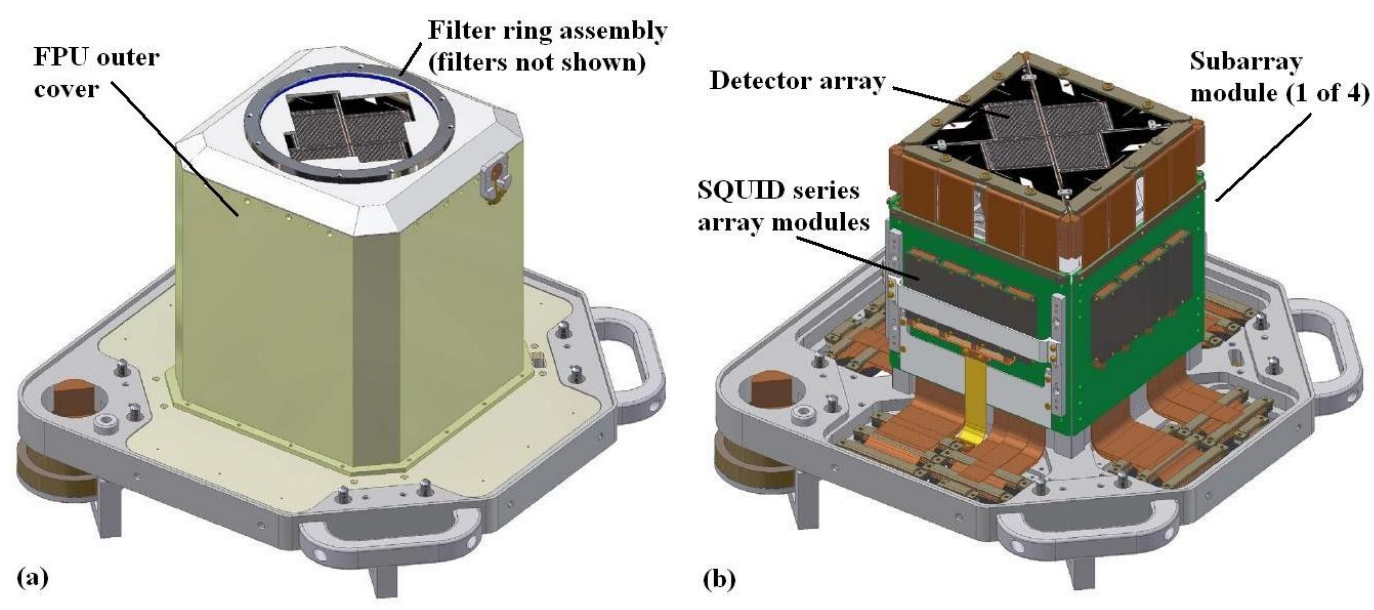

Figure 1. CAD model of the focal plane unit modules. Left: module with outer cover in place. Right: outer cover removed to expose array modules.

of high-permeability and superconducting shielding on the detector enclosure at a temperature of $\sim 1 \mathrm{~K}$. At the time this design work was carried out, the instrument cryostat had already been manufactured, and hence the shielding had to be retrofitted to the instrument. This paper goes on to describe the design and construction of the cryogenic shielding layers, and presents verification data.

\section{DESIGN OF THE SCUBA-2 INSTRUMENT AND SHIELDING LAYOUT}

The cryo-mechanical design of the SCUBA-2 instrument is described in detail elsewhere. ${ }^{4,5}$ Two focal planes of detector arrays, operating at $<100 \mathrm{mK}$, are enclosed within independent modules (known as "focal plane units", or FPUs). A CAD model of the FPUs is shown in Fig. 1. The main structure of the modules are aluminium alloy, cooled by the still of a dilution refrigerator to $\sim 1 \mathrm{~K}$. The FPUs are contained within a further enclosure (the "1-K box", see Fig. 2), also of aluminium, that provides radiation shielding and a mounting for cold optical components. Also mounted on the $1-\mathrm{K}$ box is a motor and shutter assembly to blank the detectors from incident radiation. The $1-\mathrm{K}$ box is mounted within nested radiation shields at nominal temperatures of $4 \mathrm{~K}$ and $60 \mathrm{~K}$.

Standard mu-metal has a temperature dependent performance, such that at $4 \mathrm{~K}$ the permeability of the material is approximately $10 \%$ of the ambient temperature value. While high-permeability shielding on the vacuum vessel at ambient temperature is constructed from standard mu-metal, the cryogenic shielding is composed of Metglas*, a high-permeability foil with a magnetic permeability that is only weakly dependant on temperature. Other mu-metal alloys that are designed for low-temperature shielding are available, but at considerable cost. As a foil material, Metglas was less expensive, and when fitted would add only minimally to the mass of the existing structures. The disadvantage is that Metglas is not available in large sheets, complicating the construction of large-area shields. These issues are discussed in more detail in $\S 4$.

The final shielding design recommended as a result of the modelling process consisted of a double $1 \mathrm{~mm}$ mu-metal shield on the vacuum vessel, combined with a high-permeability shield of $200 \mu \mathrm{m}$ thickness on the 1-K box and $70 \mu \mathrm{m}$ thickness on the focal plane units. An established technique for increasing the effectiveness of high-permeability shields, particularly those composed of thin foils, is to "nest" shields with several thicknesses of inert spacing materials. This approach was adopted for the cryogenic shielding design. The 1-K box and FPUs would be fitted with superconducting niobium shields in addition to the high-permeability shields.

\footnotetext{
${ }^{*}$ Metglas Inc., 440 Allied Drive, Conway, SC 29526, USA
} 


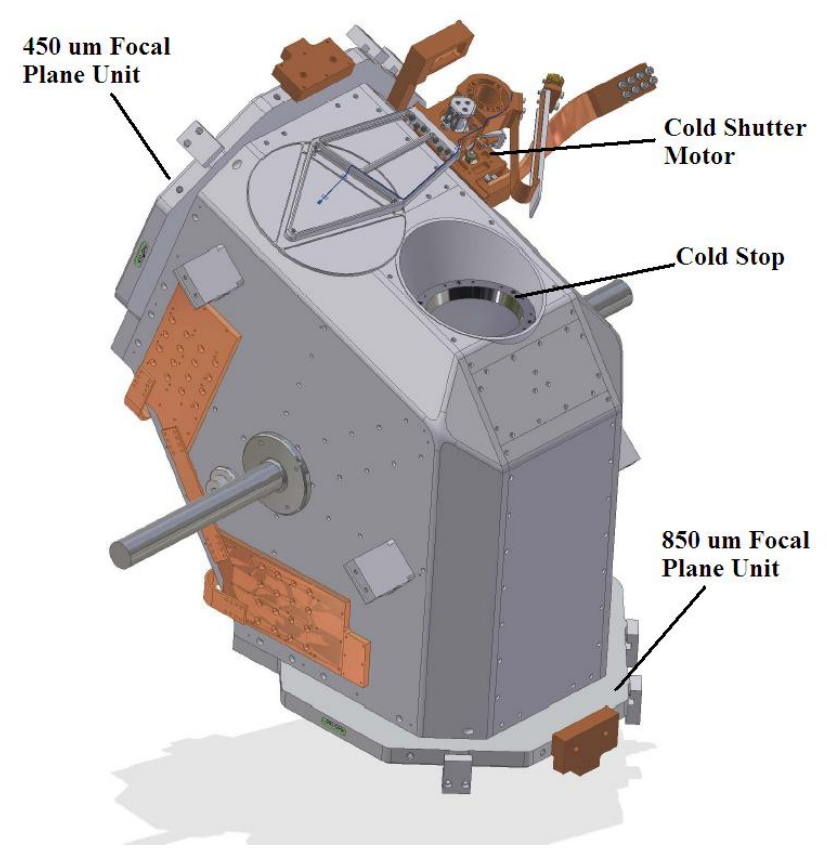

Figure 2. 3D CAD model of the $1 \mathrm{~K}$ box main structure.

\section{MODELLED SHIELDING PERFORMANCE}

The key results of the FE modelling work are summarised in Table 1, with the contribution of each stage of the shielding listed and a total attenuation factor. A more complete analysis of the overall shielding effectiveness may be found in Reference I. The effectiveness of many of the shield geometries modelled were found to have a dependance on the direction of the applied field. Since the two focal planes are orientated at right-angles in the cryostat, the focal planes have different attenuation factors. Results are therefore listed for the two focal planes individually. Furthermore, the directional dependance of the shielding effectiveness of the individual components results in a maximum and minimum total attenuation of the overall shield design. The results for both cases are detailed.

The required attenuation factor of 1500 is met by the minimum predicted figures by a reasonable margin, while the maximum predicted figures exceed the require factor by a large margin. However, it should be noted that the models used to generate the predicted figures were somewhat idealised.

\section{SHIELDING DESIGN}

The structure of the 1-K stage is composed of aluminium (6082 T6). Due to the need to control magnetic fields, it was specified that no magnetic materials (other than shielding materials) should be used in the construction of the detector enclosure. As such, screws and other fastenings in the structure are of brass or aluminium rather than steel. The only exception to this are the screws used to make the copper-copper joints for the thermal straps. The need to produce a good contact force on these joints required stainless steel screws rather than softer metals. To minimise the effect of the presence of steel in the structure, A2 grade (non-magnetic) stainless screws were used.

The key challenges in the construction of the shielding for the 1-K box were how to efficiently cover the large area of the box with Metglas and niobium foil, how to attach the small sections of foil to the aluminium structure, and, to a lesser extent, how to produce a nested Metglas shield.

The nominal dimensions of Metglas 2705M foil are 2 inches $(50.8 \mathrm{~mm})$ wide and $0.85 \mathrm{mil}(21.6 \mu \mathrm{m})$ thick. In order to maintain continuity in the layer, adjacent sections of foil must be overlapped by at least 0.75 inches 
Table 1. Summary of contributions from components of the shielding design to the overall attenuation of external magnetic fields. Attenuation factors are listed for individual shielding stages making up the the maximum and minimum total attenuation. The variation in the contributions of the different shielding stages is the result of a dependance in shielding effectiveness on the direction of the applied field (see text for further discussion).

\begin{tabular}{lcc}
\hline \hline Shielding & \multicolumn{2}{c}{ Attenuation factor } \\
\cline { 2 - 3 } & $850 \mu \mathrm{m}$ focal plane & $450 \mu \mathrm{m}$ focal plane \\
\hline Maximum total attenuation: & 2.5 & 2.5 \\
Vacuum vessel mu-metal shield & 10 & 10 \\
1-K box high-permeability shield & 40 & 40 \\
1-K box superconducting shield & 4 & 10 \\
Focal plane unit high-permeability shield & 10 & 2 \\
Focal plane unit superconducting shield & 40000 & 20000 \\
\hline Total attenuation with superconducting shield & & \\
\hline Minimum total attenuation: & 2.5 & 2.5 \\
Vacuum vessel mu-metal shield & 30 & 30 \\
1-K box high-permeability shield & 4 & 4 \\
1-K box superconducting shield & 10 & 10 \\
Focal plane unit high-permeability shield & 2 & 6000 \\
Focal plane unit superconducting shield & 6000 & \\
\hline Total attenuation & & \\
\hline \hline
\end{tabular}

(19 mm). ${ }^{6}$ Attachment of the large number of Metglas foil strips required to cover the box area would be impossible with screws due to the large number of holes that would be required. Metglas foil had already been used extensively to shield the SCUBA-2 subarray test cryostat at Cardiff University; ${ }^{7}$ the method of attachment used for the foil strips was a combination of Kapton and Mylar tape. Concerns over the long-term reliability of this method made this an unsuitable approach for the instrument shielding. The method ultimately selected to attach the shielding was to bond the Metglas foil onto a backing material using epoxy, such that the completed sheets could be cut to fit the box structure. The details of this process are described below. Use of epoxy was also a useful way to thermally sink the shielding material to ensure the layers cooled to the stage temperature.

The FE modelling results for the $1-\mathrm{K}$ box required a Metglas shield of at least $200 \mu \mathrm{m}$ total thickness. The method recommended by the manufacturers for foil shields suggests that foil layers are spaced by 3 to 4 thicknesses of an inert material, although FE modelling of nested foil shields had previously indicated that shields are effective with up to 10 thicknesses of space between layers. The backing sheet used for the majority of the shielding was aluminised Mylar with a thickness of $127 \mu \mathrm{m}$, or approximately 6 thicknesses of Metglas. Mylar was selected as a backing material due to ready availability in a number of different thicknesses, and suitability for use in a vacuum. The shielding could in principle be made as a single sheet and attached to the 1-K box using a small number of screw fixings. Tests carried out during development identified Stycast $2850 \mathrm{FT}^{\dagger}$ as a suitable adhesive to bond the Metglas foil to Mylar sheets. A test section of the composite shielding is shown in Fig. 3.

The shielding produced for the 1-K box consisted of an inner Metglas layer on a $15 \mu \mathrm{m}$ aluminised Mylar backing, a middle sheet of Metglas on $127 \mu \mathrm{m}$ Mylar, and an outer sheet of Metglas that also incorporated the niobium shield. This outermost layer was composed of a $127 \mu \mathrm{m}$ Mylar backing sheet with Metglas foil, then tiles of $0.25 \mathrm{~mm}$ niobium bonded to the Metglas, and a final layer of $127 \mu \mathrm{m}$ Mylar for the outer surface. These shielding layers were produced as oversized sheets, then cut to size using a net of the 1-K box structure. Screw holes matching the pattern in the aluminium structure were pre-drilled. Larger apertures for thermal contacts were cut either by hand or using hole saws.

\footnotetext{
${ }^{\dagger}$ Emerson and Cuming, 46 Manning Road, Billerica, MA 01821, USA (http://www.emersoncuming.com)
} 


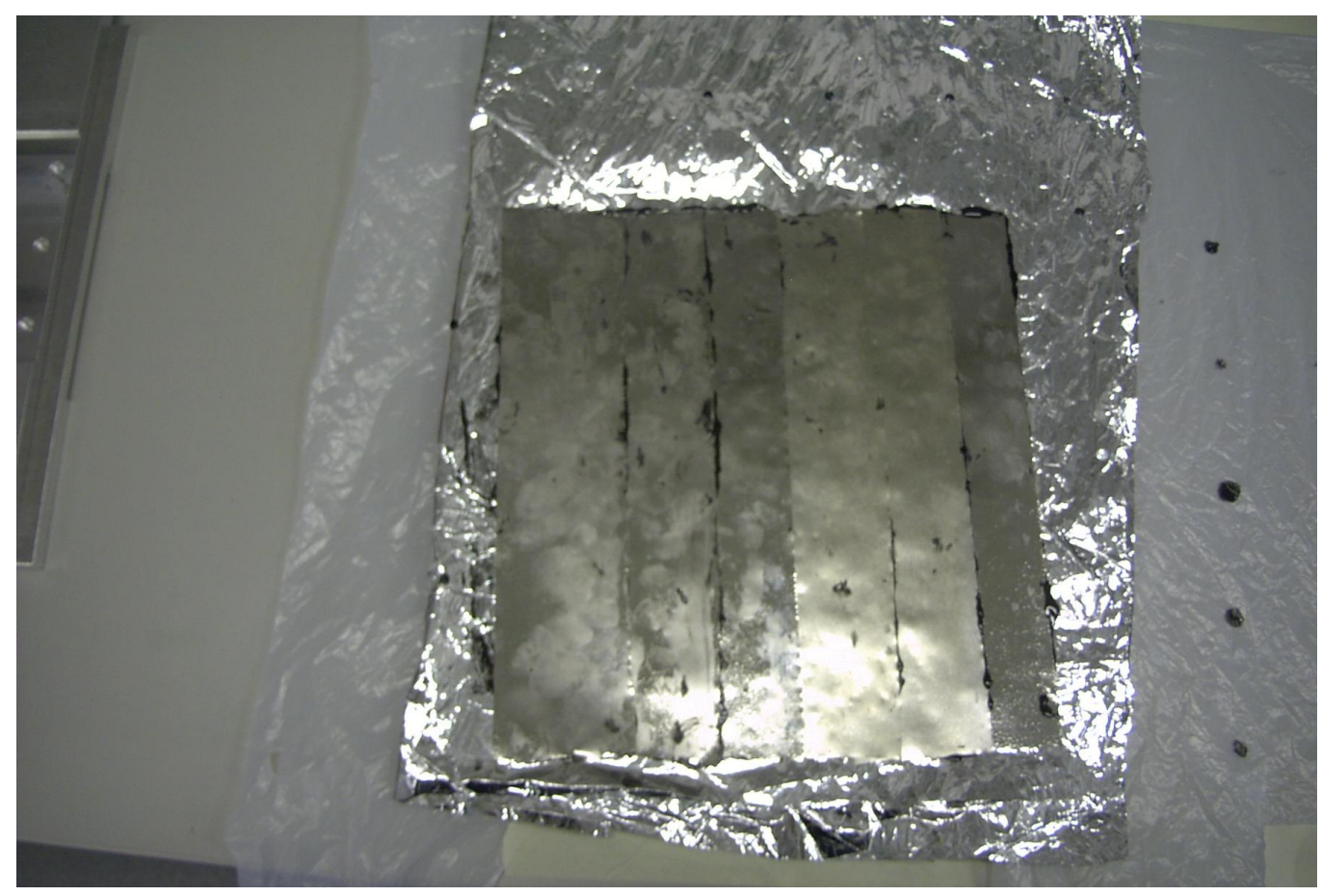

Figure 3. Section of Metglas composite sheet. Approximately 10 strips of Metglas are bonded to a backing sheet of 15 $\mu \mathrm{m}$ thick aluminised Mylar using Stycast 2850FT epoxy.

The shielding sheets were attached to the aluminium box primarily by a pattern of M3 brass screws around the edges of the shielding. The pre-cut through-holes for the screws were intentionally made oversized, so stacks of brass washers were used to ensure a good contact between the screw heads and shielding layers. Only a small number of screw fixings were used for the layers to reduce the number of holes required in the 1-K box, so to improve the thermal contact between the shielding and aluminium strips of double-sided adhesive copper tape were placed between the shielding layers. Finally, the loose edges of the shielding were sealed with Mylar tape.

The original intention had been to fold the majority of each shielding layer onto the aluminium structure as a single section, but due to limited flexibility of the thicker sheets, this proved difficult. To overcome this, the two outermost layers were separated into a series of flat panels, which were then attached independently to the 1-K box. Continuity between the panels was maintained by taping additional strips of Metglas along the joints using copper and Mylar tape, again maintaining a minimum overlap of $20 \mathrm{~mm}$.

Thermal contact to the 1-K box structure to the wick from the dilution refrigerator still consists of four large copper plates attached to the aluminium panels by a pattern of screws and a large-area epoxy joint. The need to make contact directly to the aluminium forced large gaps to be left in the shielding layers. To reduce the penetration of flux through the the shielding, in particular the superconducting layer, oversized panels of niobium foil were attached inside the box in the area of the copper plates.

The shielding on the focal plane units was attached to the removable outer covers that fit over the detector subarrays. The shielding consisted of three layers of Metglas foil attached by adhesive tape to the aluminium covers, with a $20 \mathrm{~mm}$ overlap between adjacent strips of foil. Sections of $0.25 \mathrm{~mm}$ thick niobium foil were then fitted over the Metglas using M2.5 brass screws. Finally, joints between the niobium foil sections and the edges of the layer were sealed with aluminium adhesive tape. Fig. 4 shows one of the focal planes with the outer cover in place over the detectors.

The final shielding differed from the models in that the structure of the 1-K box forced more discontinuities in the shielding than had originally been anticipated. This was particularly true for the focal plane units, which 


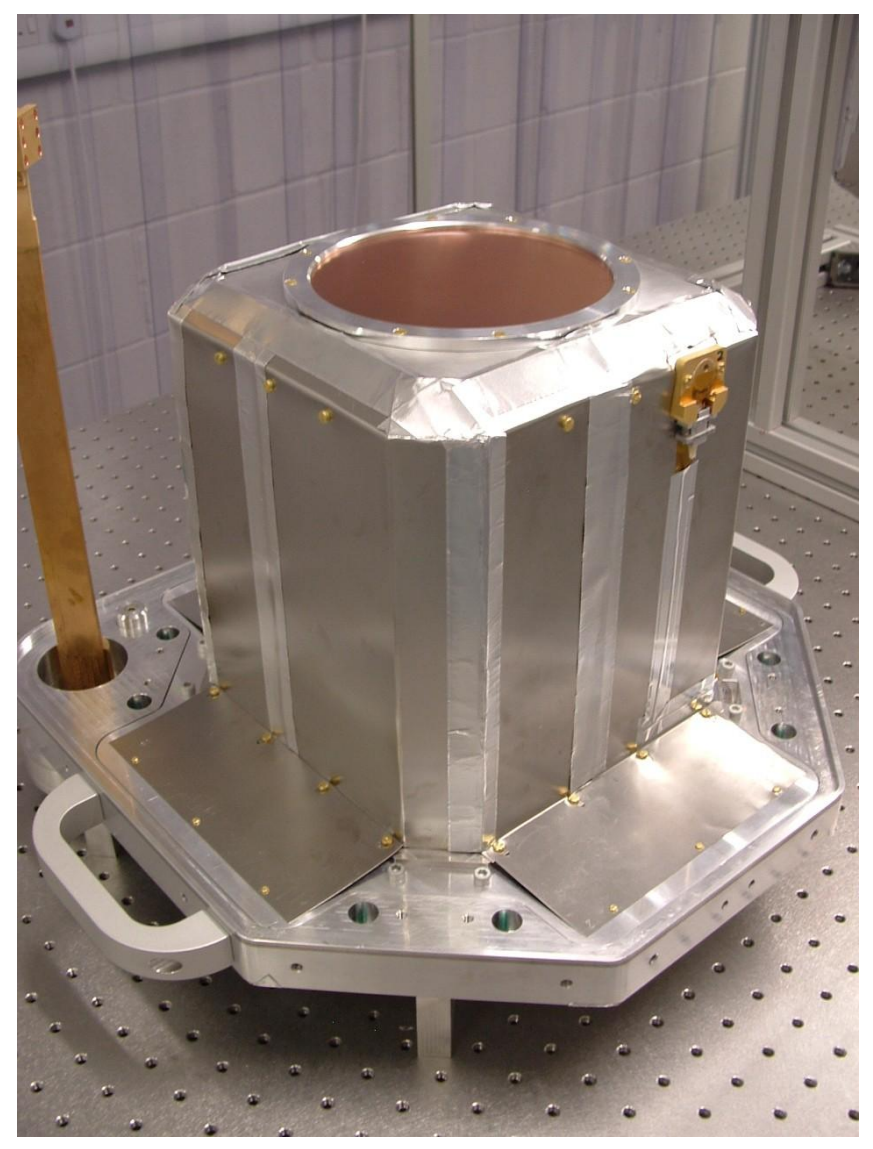

Figure 4. Focal plane unit with the shielded outer cover in place.

due to the design of the end plates, were no longer completely enclosed shells as had been modelled. It is likely that this has a detrimental effect on the shielding performance by allowing flux to leak into the enclosed volume, reducing the effectiveness of the $1-\mathrm{K}$ and FPU shields. Counter to this, the high-permeability shielding on the 1-K box used larger spacing layers between the Metglas foil than included in the model. The modelled shielding for the $1-\mathrm{K}$ stage had a total thickness of $200 \mu \mathrm{m}$, whereas the final shield was approximately $450 \mu \mathrm{m}$ thick. Furthermore, the vacuum vessel shielding was constructed such that the mu-metal formed a complete shell, as opposed to the model which had gaps between shield sections. These latter changes would serve to increase the shielding effectiveness.

Additional shielding was applied to the motor for the cold shutter. Although the motor used for the mechanism was designed to have a low magnetic moment, simple dipole calculations indicated that the field at the detectors due to the motor was too large. The motor assembly was wrapped with a 10 layer shield of Metglas foil, with a niobium foil section fitted between the motor and the 1-K box structure.

\section{SHIELDING VERIFICATION}

The effectiveness of the magnetic shielding was determined by applying an external field from an electromagnet, and measuring the response of the second stage SQUIDs (SQ2s). The SQ2s were used since, in principle, they should be the most sensitive part of the readout chain to changes in the magnetic environment. The first stage SQUIDs have additional shielding effects due to the presence of the superconducting detector ground plane, while the series array SQUIDs are shielded by independent superconducting and high-permeability cans. Furthermore, the output from the readout chain is complicated since the signal is actually a convolution of the response of 
each device in the chain, making interpretation and calibration of the response to an external field complex. By using the SQ2s, the output is a convolution of the SQ2 and the SSA response. Making the reasonable assumption that the SSAs are unaffected by the external field, the output essentially measures the SQ2 response only. Measurements were made on the $850 \mu \mathrm{m}$ array only, since the $450 \mu \mathrm{m}$ array was unavailable during the tests. From the predicted shield performance, the attenuation of fields at the $850 \mu \mathrm{m}$ focal plane is as much as a factor 2 higher than the $450 \mu \mathrm{m}$ focal plane. The measurements therefore represent an upper limit on the effectiveness of the shielding.

Measurements of the field response were made with several magnets of varying strength, and placed at different locations around the cryostat. The field stength was measured using a gaussmeter at a distance of $10 \mathrm{~cm}$. This is approximately the distance between the magnet and the cryostat shielding layer, with the magnetic held external to the cryostat. With the weaker magnets (applying fields of $\sim 0.1$ and $\sim 0.3 \mathrm{mT}$ ), no response was seen on the SQUID output. It was necessary to use a larger magnet, with a field of $\sim 1 \mathrm{mT}$, before a response was observed. The field was applied in several different directions and locations around the cryostat. The strongest response was seen with the magnet at the backshell of the cryostat, in the location physically closest to the arrays, and with the field applied perpendicular to the $850 \mu \mathrm{m}$ focal plane. With the magnet in other locations, little or no response was observed.

The response of a selection of SQ2s with the magnet close to the focal plane is shown in Fig. 5. The data is taken as a timestream, with the output from the readout chain measured in DAC units. The three plots shown are from columns 3,16 and 30 (of 32 columns in a subarray); these were selected since the channels showed a clear response to the field change (some channels were very noisy, making the response harder to measure, or were simply not operational), and since the distribution of these channels indicates the change in response across the array.

To linearise the response of the SQUIDs, the devices are operated with a feedback applied to the superconducting loop as a magnetic field from a coil. ${ }^{8}$ A change in the field at the SQUID may therefore be induced by changing the current in the coil. The SQUID output will respond in the same way to a field change due to the application of an external field as to a change in the current in the feedback coil. The response of the SQUID to the external field may therefore be calibrated by making a deliberate change of 100 DAC units to the SQ2 feedback, and measuring the change in output. Each SQUID will not have exactly the same response, so the same feedback change was made on all channels to give an independent calibration for each channel. The calibration for channels 3, 16 and 30 are also shown in Fig. 5.

The output change in response to the external field can be compared to the response to the change in feedback current, resulting in a feedback change equivalent to the change due to the external field. The response to the SQUID to changes in feedback or magnetic field strength is periodic. The characteristic response (a $V-\phi$ curve) has a period of one quantum of flux, $\Phi_{0}$. The SQUIDs for the SCUBA-2 detectors are designed such that the full range of the feedback (65000 DAC units) sweeps out a full $V(\phi)$ cycle. Hence, we can obtain the change in field at the SQ2s, $\delta B$, in units of $\Phi_{0}$, due to the external field by taking the feedback change equivalent to the presence of the external field, $\Delta$ as a fraction of the full range, such that

$$
\delta B=\frac{\Delta}{65000} \Phi_{0}
$$

One flux quantum is approximately equal to $2 \times 10^{-15} \mathrm{Tm}^{2}$. This is a measure of the magnetic flux enclosed by a superconducting loop; taking the area of the SQUID to be $10 \mu \mathrm{m}$ square, $\delta B$ may be converted to a field change in Tesla. Comparison to the applied field of $\sim 1 \mathrm{mT}$ gives the attenuation factor. The calculation for the three channels in Fig. 5 using this method is summarised in Table 2.

The calculated attenuation factors for these channels range from 50000 to 100000 . This is broadly consistent with the high-end predicted attenuations from the FE models (see Table 1), although the measured attenuations are slightly greater than the predicted figures. It would be expected that the measured performance would be lower than the modelled performance, since in many respects the models were idealised. The major differences between the shielding models and the final construction were discussed previously. Some of the differences, such 
Channel 3 Magnet

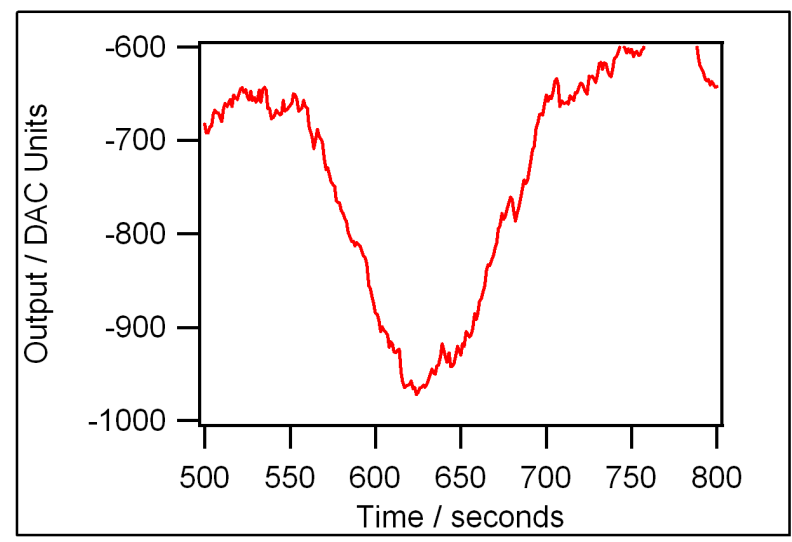

Channel 16 Magnet

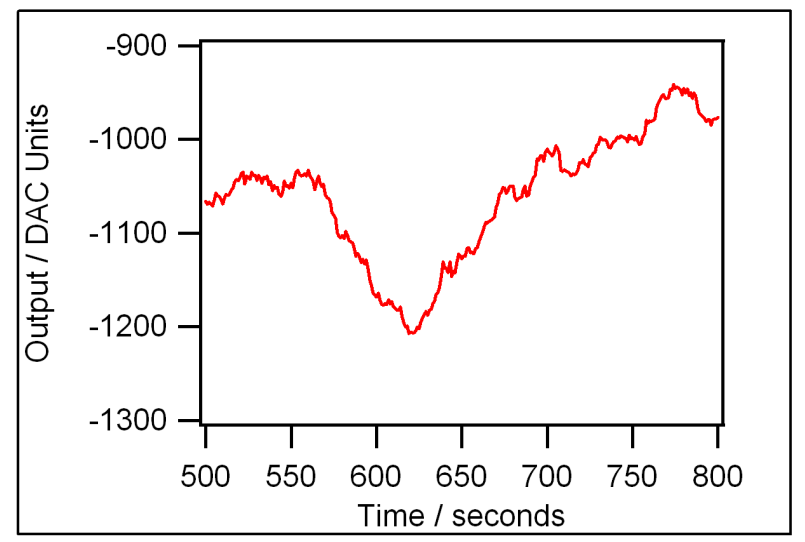

Channel 30 Magnet

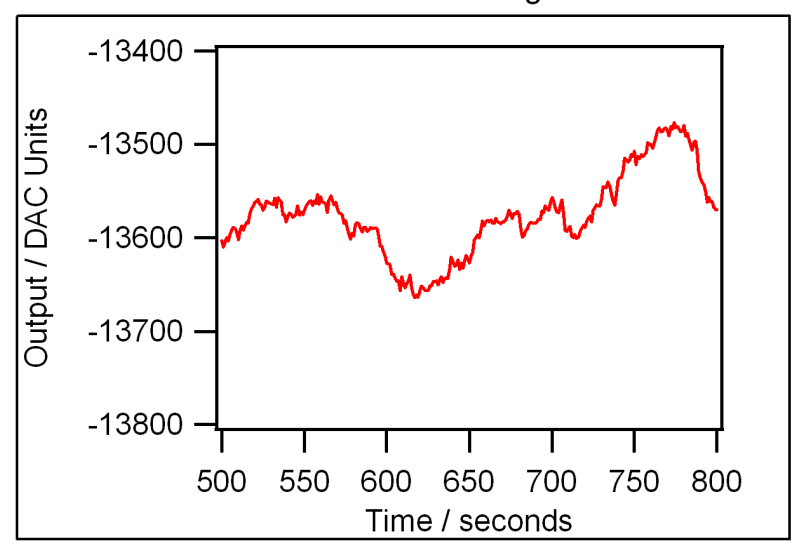

Channel 3 Calibration

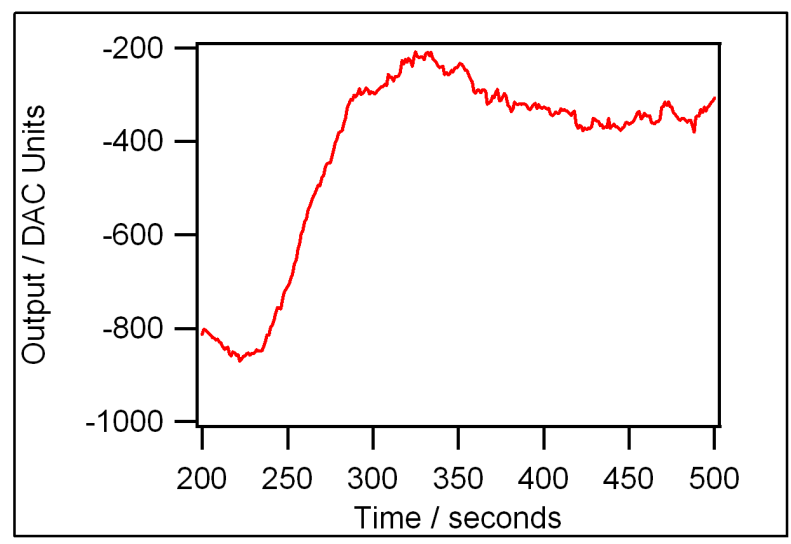

Channel 16 Calibration

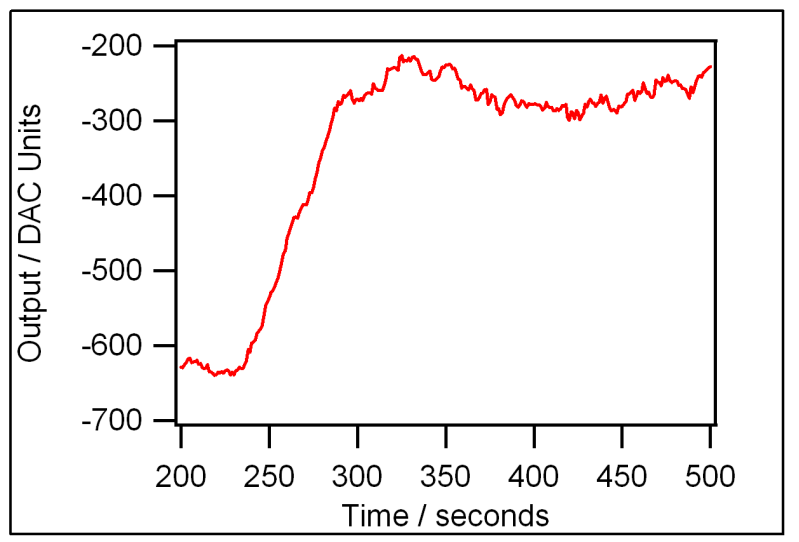

Channel 30 Calibration

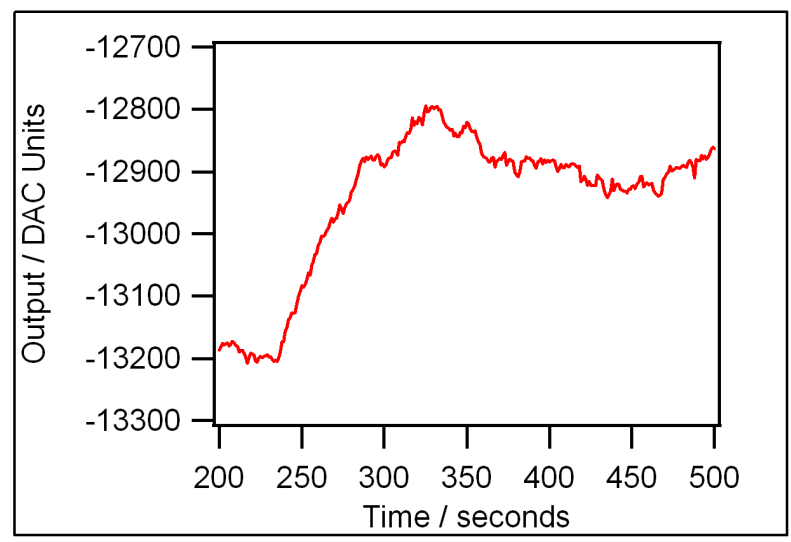

Figure 5. Time series plots of detector output on channels 3, 16 and 30 of an $850 \mu$ m subarray. The plots in the left column show the response of the second stage SQUIDs to an external magnetic field of $\sim 1 \mathrm{mT}$, with the magnetic switched on at $\sim 550$ seconds. The plots on the right show the response of the same SQUIDs to a change of 100 DAC units on the SQ2 feedback, allowing calibration of the response to the external field. The feedback change was applied at $\sim 250$ seconds. See text for discussion of the calibration method and the analysis of the response. 
Table 2. Calculated external magnetic field attenuations for channels 3,16 and 30 on an $850 \mu$ m subarray. The intermediate steps of the calculations following the method described in the text are also included.

\begin{tabular}{lccc}
\hline \hline & \multicolumn{3}{c}{ Channel Number } \\
\cline { 2 - 4 } & 3 & 16 & 30 \\
\hline Response to external field / DAC units & 300 & 250 & 100 \\
Response to feedback change / DAC units & 600 & 400 & 400 \\
$\delta B / \Phi_{0}$ & $8 \times 10^{-4}$ & $1 \times 10^{-3}$ & $4 \times 10^{-4}$ \\
$\delta B / \mathrm{nT}$ & $16^{-4}$ & 19 & 8 \\
\hline Attenuation & $6 \times 10^{4}$ & $5 \times 10^{4}$ & $1 \times 10^{5}$ \\
\hline \hline
\end{tabular}

as discontinuities in the shields would tend to decrease the actual shielding effectiveness, while others, such as the thicker shield layers on the 1-K box, would be expected to increase the attenuation of the shields.

The attenuation factors for channels 3 and 16 are similar, increasing slightly for channel 30 . With channel 3 on the outer edge and channel 30 at the centre of the focal plane, this may indicate a field gradient across the focal plane, as expected from the modelling of the detector array groundplane. This is not conclusive. The model results predict a change of approximately an order of magnitude in the field strength between the edge of the focal plane and the centre, whereas the measured results imply a factor 2 difference. Without data from other subarrays on the focal plane, the presence of a gradient can not be reliably shown. The high attenuation factor measured for these channels would indicate that even if there is a field gradient, it is sufficiently small that it will not affect the performance of the arrays.

As discussed previously, the overall attenuation for the $850 \mu \mathrm{m}$ focal plane may be as much as a factor two greater than for the $450 \mu \mathrm{m}$ focal plane, as predicted from the model results. However, the predicted $450 \mu \mathrm{m}$ results are still within the specified requirement for the field attenuation.

\section{CONCLUSIONS}

This paper has described the design, construction and testing of the cryogenic magnetic shielding for the SCUBA2 instrument. The cryogenic shielding was fitted to the coldest stages of the cryostat, using a novel composite technique of bonding Metglas high-permeability material, niobium superconducting shielding and Mylar spacing to cover large areas of the instrument structure at a temperature of $\sim 1 \mathrm{~K}$. Combined with ordinary highpermeability shielding at ambient temperature, the measured attenuation of an external magnetic field at the detectors was determined to be between $5 \times 10^{4}$ and $1 \times 10^{5}$, which compares well with the upper limit of the performance predicted from finite-element modelling. The measured values are well within the specified minimum field attenuation factor of 1500 .

Although measurements could only be made on one of the two instrument focal planes, the modelling results indicated that the attenuation on the second focal plane would be at most a factor 2 lower than these values. This is also within the specified attenuation.

\section{ACKNOWLEDGMENTS}

The authors would like to thank David Montgomery and Mark Cliffe of the UK Astronomy Technology Centre for input to the shielding development process.

MIH is supported by a Science and Technology Facilities Council studentship.

The SCUBA-2 project is funded by the UK Science and Technology Facilities Council, the JCMT Development Fund and the Canadian Foundation for Innovation. 


\section{REFERENCES}

1. W. Holland, M. MacIntosh, A. Fairley, D. Kelly, D. Montgomery, D. Gostick, E. Atad-Ettedgui, M. Ellis, I. Robson, M. Hollister, A. Woodcraft, P. Ade, I. Walker, K. Irwin, G. Hilton, W. Duncan, C. Reintsema, A. Walton, W. Parkes, C. Dunare, M. Fich, J. Kycia, M. Halpern, D. Scott, A. Gibb, J. Molnar, E. Chapin, D. Bintley, S. Craig, T. Chylek, T. Jenness, F. Economou, and G. Davis, "SCUBA-2: a 10,000-pixel submillimeter camera for the James Clerk Maxwell Telescope," in Millimeter and Submillimeter Detectors and Instrumentation for Astronomy III., J. Zmuidzinas, W. S. Holland, S. Withington, and W. D. Duncan, eds., Proceedings of the SPIE 6275, p. 62751E, July 2006.

2. R. P. Welty and J. M. Martinis, "A series array of dc SQUIDs," IEEE Transactions on Magnetics 27, p. 2924, Mar. 1991.

3. M. I. Hollister, M. D. Audley, W. D. Duncan, and W. S. Holland, "Finite-element modeling of magnetic shielding for SCUBA-2," in Millimeter and Submillimeter Detectors and Instrumentation for Astronomy III., J. Zmuidzinas, W. S. Holland, S. Withington, and W. D. Duncan, eds., Proceedings of the SPIE 6275, p. 627522 , July 2006.

4. D. Gostick, D. Montgomery, B. Wall, H. McGregor, M. Cliffe, A. Woodcraft, and F. Gannaway, "The cryomechanical design of SCUBA-2: a wide-field imager for the James Clerk Maxwell Telescope," in Ground-based Instrumentation for Astronomy., A. F. M. Moorwood and I. Masanori, eds., Proceedings of the SPIE 5492, p. 1743, Sept. 2004.

5. A. L. Woodcraft, F. C. Gannaway, D. C. Gostick, and D. Bintley, "Thermal design of the SCUBA-2 instrument detector stage and enclosure," in Astronomical Structures and Mechanisms Technology., J. Antebi and L. Dietrich, eds., Proceedings of the SPIE 5498, p. 446, Oct. 2004.

6. "MuShield Magnetic Shielding Catalogue and Design Guide." available from The MuShield Company, 9 Ricker Avenue, Londonderry, New Hampshire 03053, USA (http://www.mushield.com).

7. A. L. Woodcraft, P. A. R. Ade, D. Bintley, J. S. House, C. L. Hunt, R. V. Sudiwala, W. B. Doriese, W. D. Duncan, G. C. Hilton, K. D. Irwin, C. D. Reintsema, J. N. Ullom, M. D. Audley, M. A. Ellis, W. S. Holland, M. Macintosh, C. C. Dunare, W. Parkes, A. J. Walton, J. B. Kycia, M. Halpern, and E. Schulte, "Electrical and optical measurements on the first SCUBA-2 prototype 1280 pixel submillimeter superconducting bolometer array," Review of Scientific Instruments 78, p. 4502, Feb. 2007.

8. J. A. Chervenak, K. D. Irwin, E. N. Grossman, J. M. Martinis, C. D. Reintsema, and M. E. Huber, "Superconducting multiplexer for arrays of transition edge sensors," Applied Physics Letters 74, p. 4043, June 1999. 